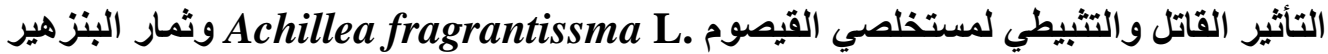 Citrus aurantifolia L.

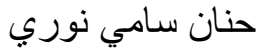 فرع العلوم التمريضية الأساسية / كلية التمريض / جامعة الموصل / العراق ـ
}

\section{الخلاصة}

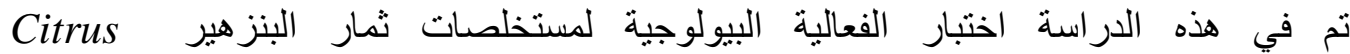

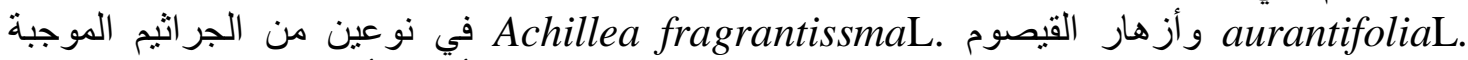
لصبغة كرام Bacillus subtitlis و و و Staphylococcus aureus وأربعة أنواع من الجر اثيم السالبة

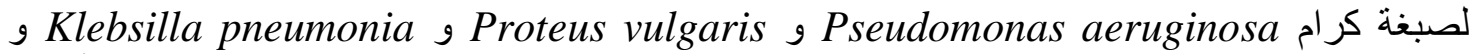
Escherichia coli

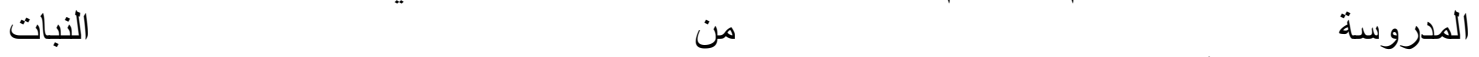

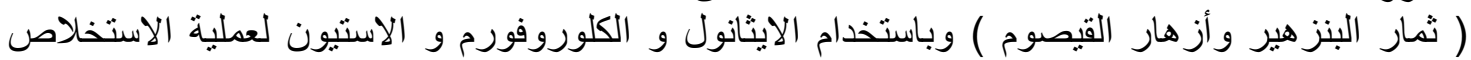

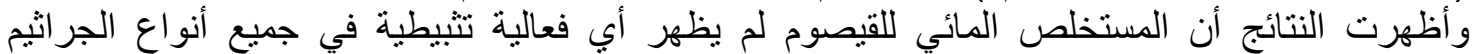

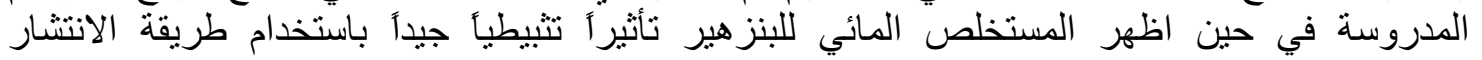

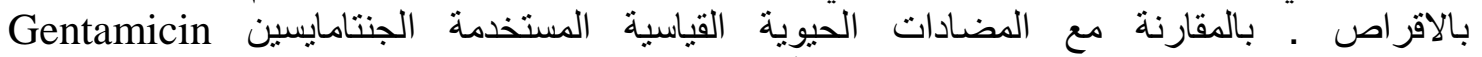

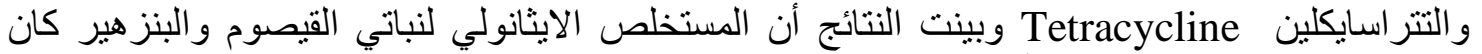

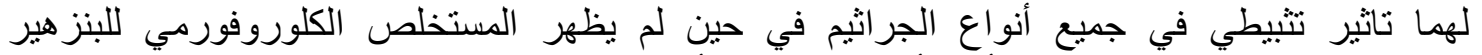

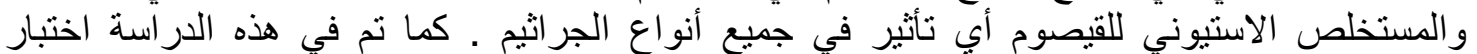

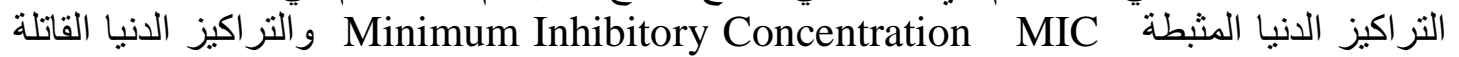
Minimum Bactericidal Concentration MBC

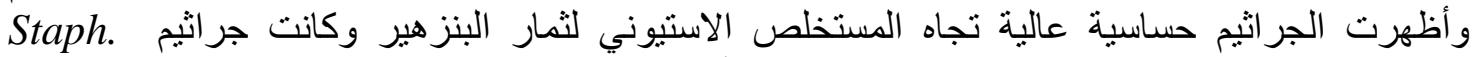

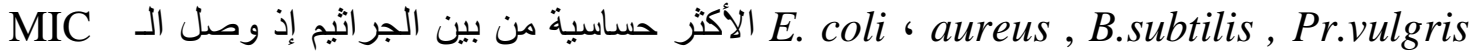

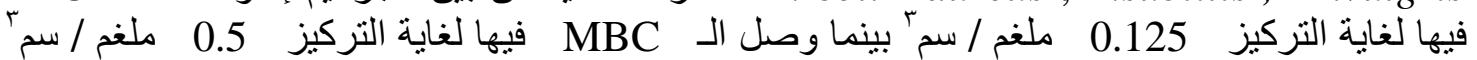

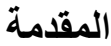

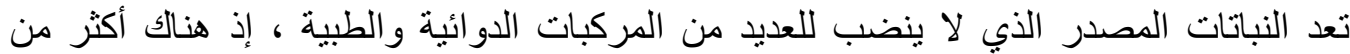

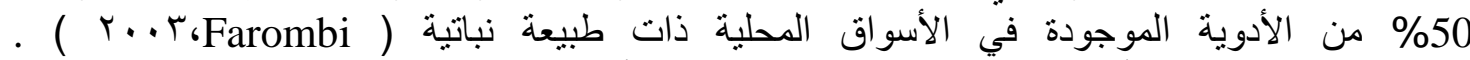

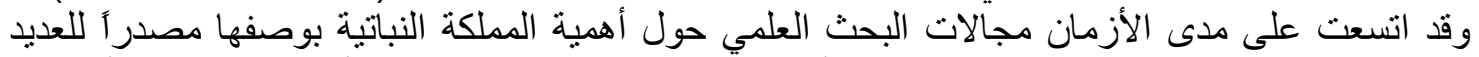

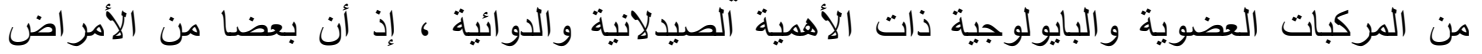

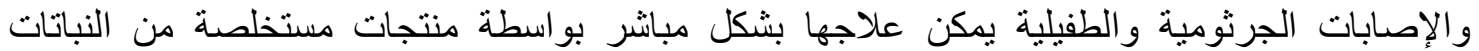

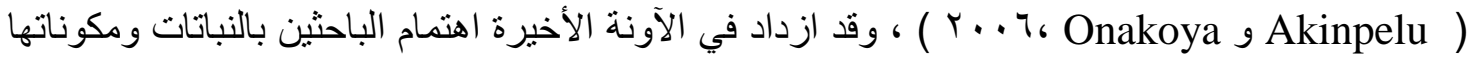

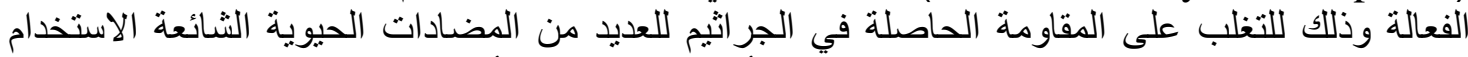

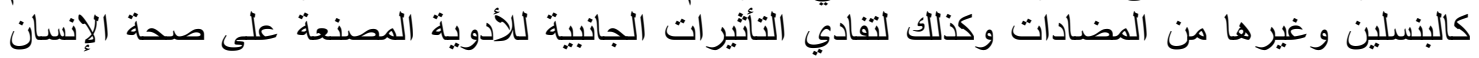

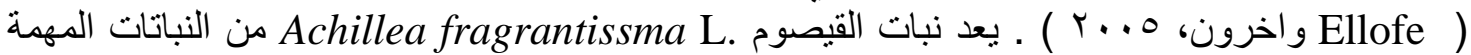

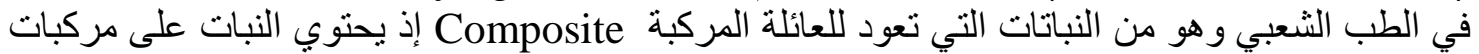

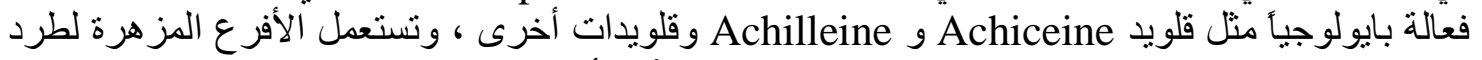

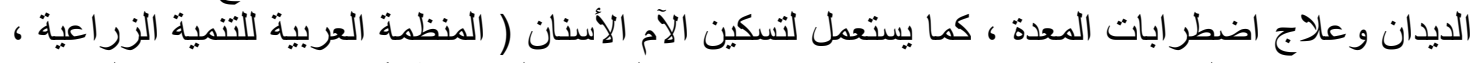

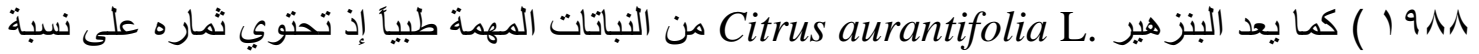

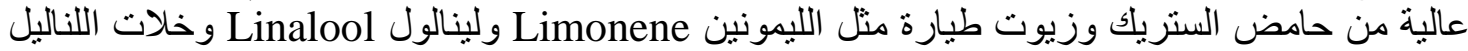
Linalyl acetate بوصفها مقويأ للمعدة وضد مرض الإسقربوط ( طلاس ، 199 1) . 


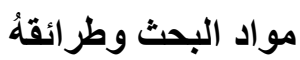

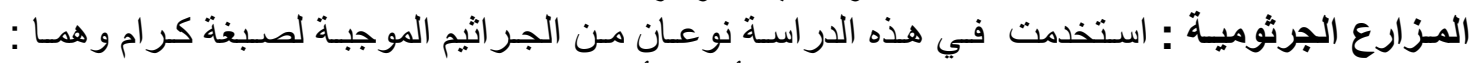
Bacills subtilis ، staphylococcus aureus Pseudomonas aeruginosa, Proteus vulgaris, Klebsilla pneumonia, Escherichia

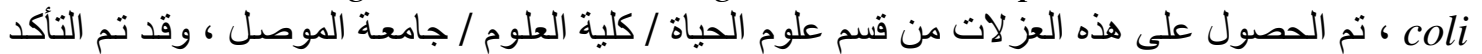

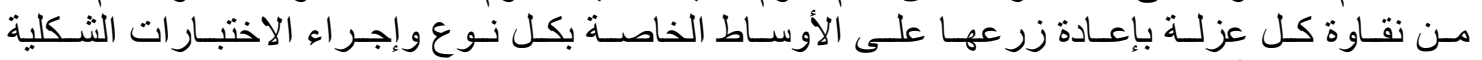

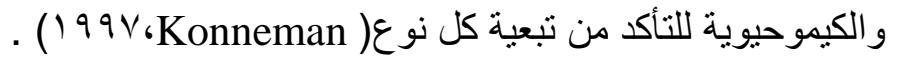

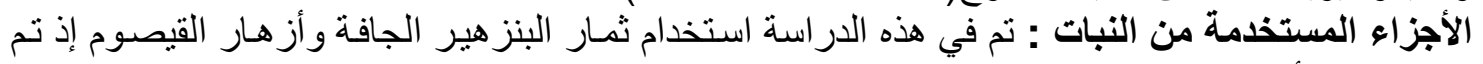

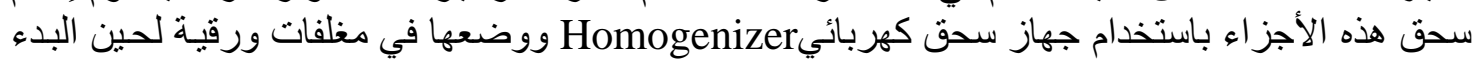

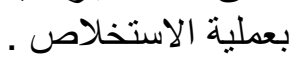
تحضير المستخلصات النباتية : تصنية

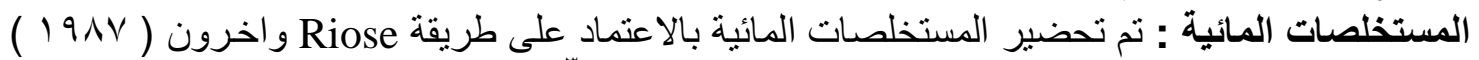

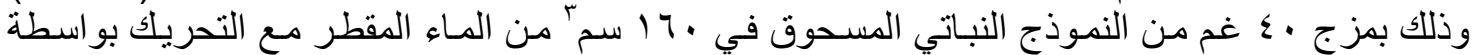

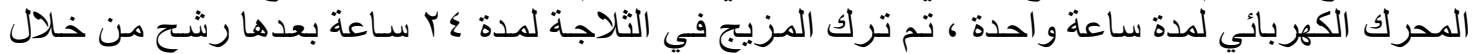

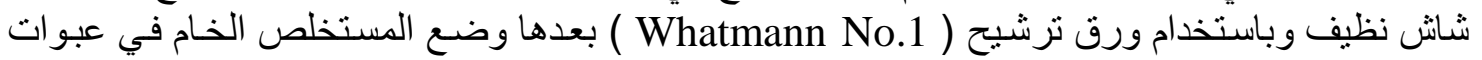

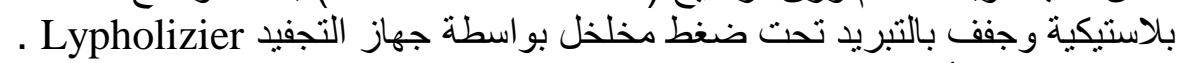

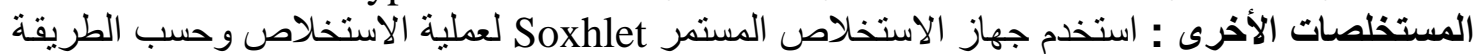

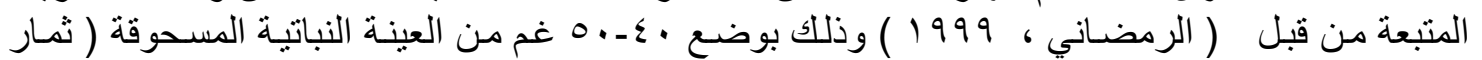

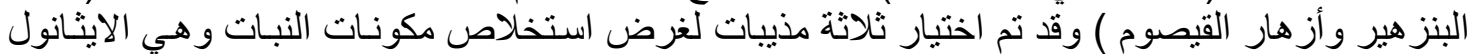

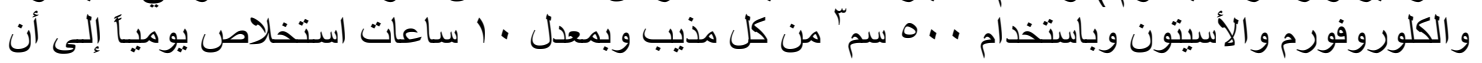

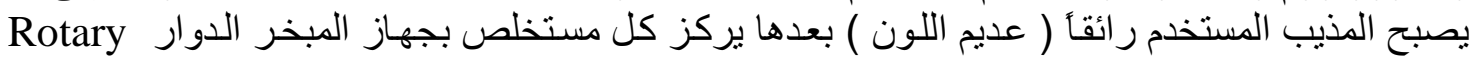
من Vaccum Evaporator من المستخلص وتختبر فعاليتها المضادة للجر اثيم.

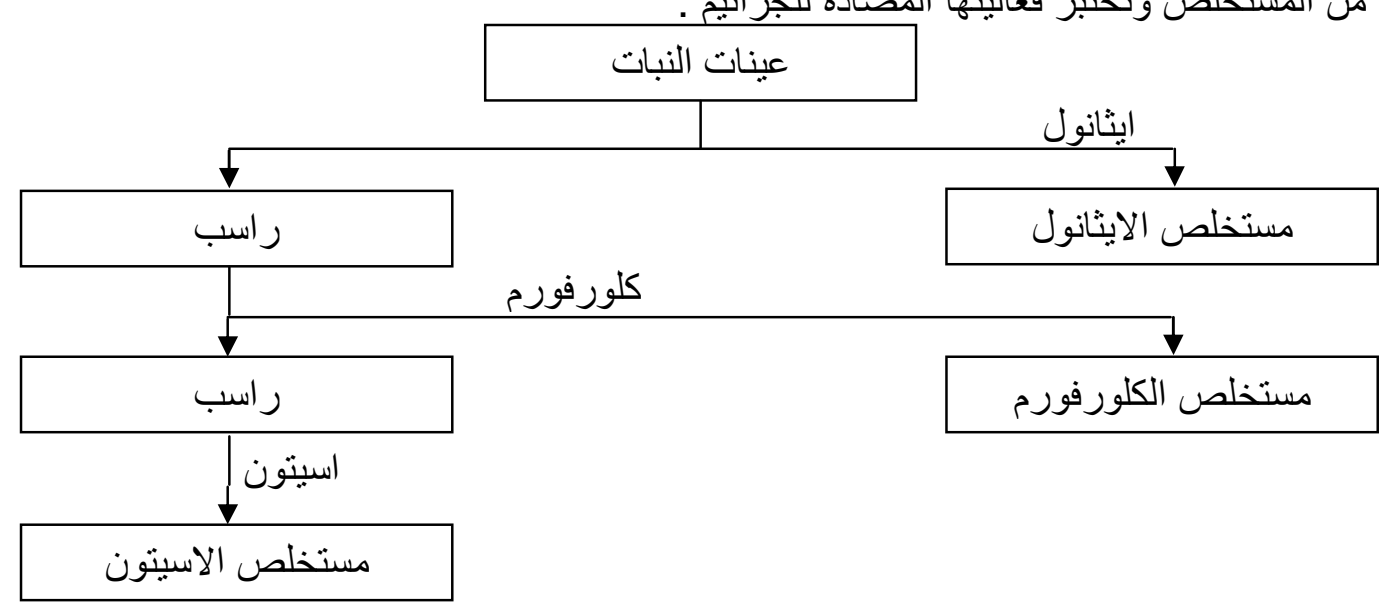

Soxhlet الثكل ( 1 ) : خطوات الاستخلاص بجهاز

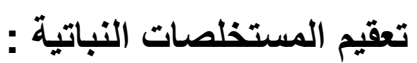
تعقيم المستخلصات المائيةً : اخذ ا غم من المستخلص المائي الجاف و أذيب في ه سمّ من الماء المقطر

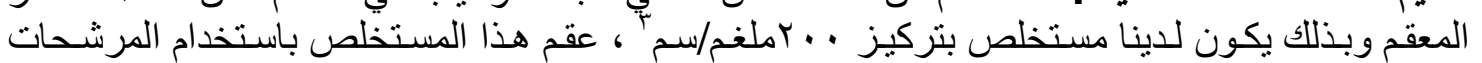

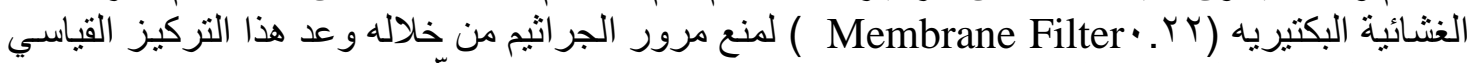

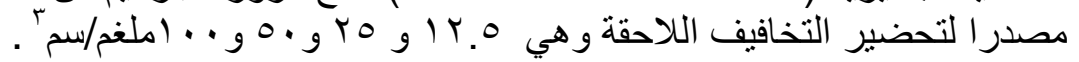


تعقيم المستخلصـات الأخرى : حضر المستخلص الايثنانولي و الكلورفورمي و الاسيتوني عن طريق إذابـة

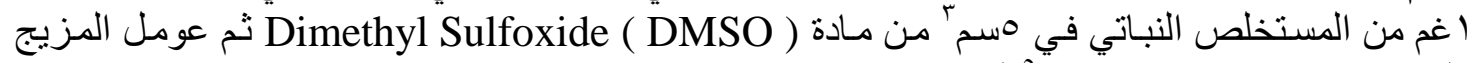

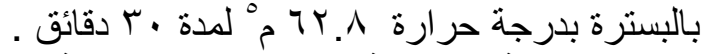

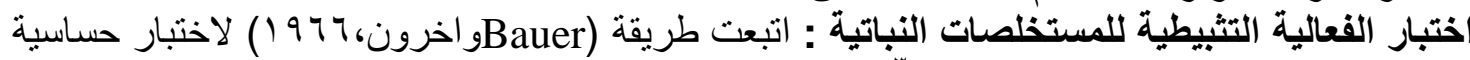

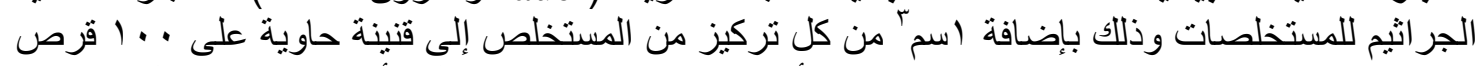

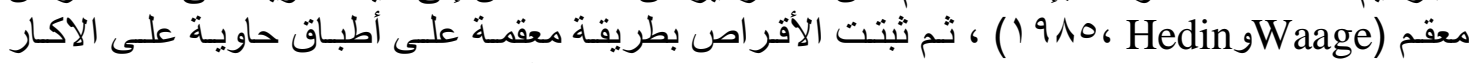

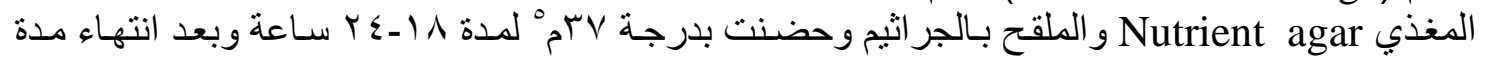

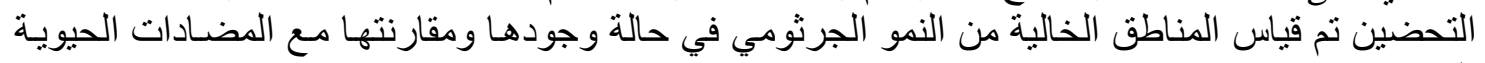

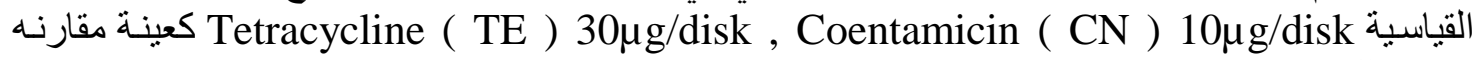
موجبة للجر اثيم .

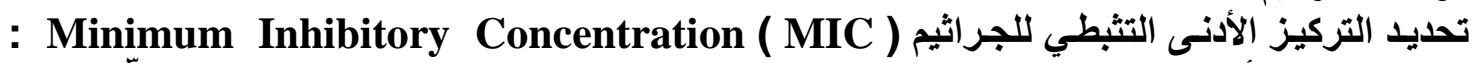

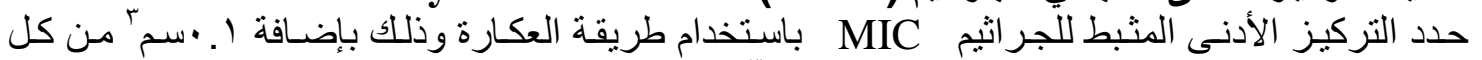

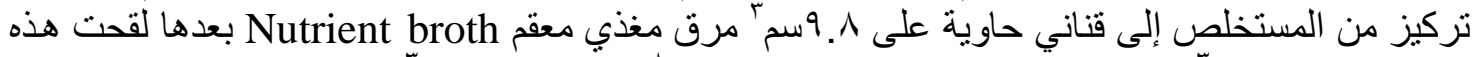

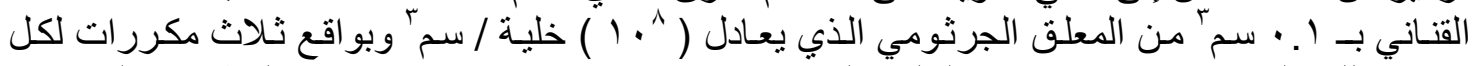

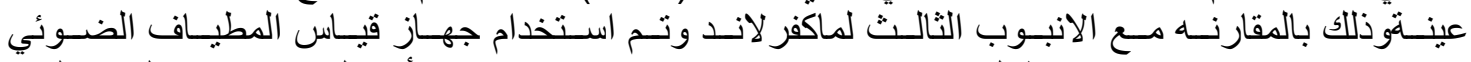
Spectrophotometer

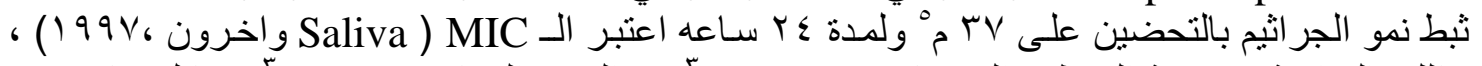

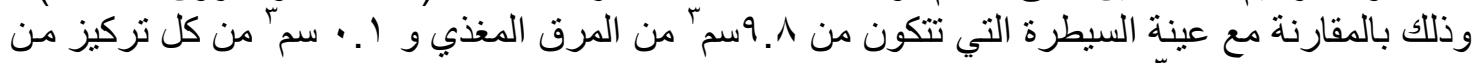

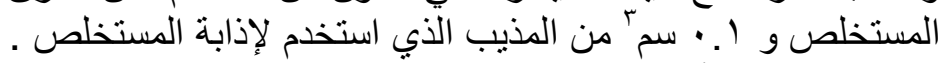

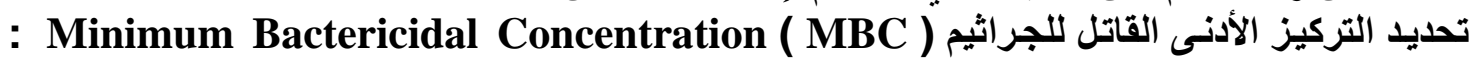

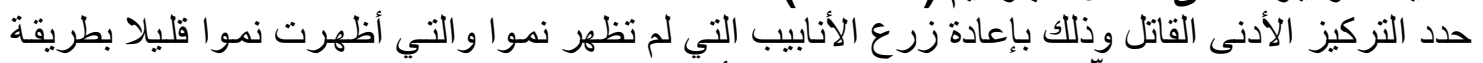

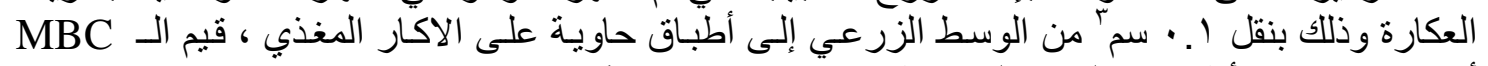

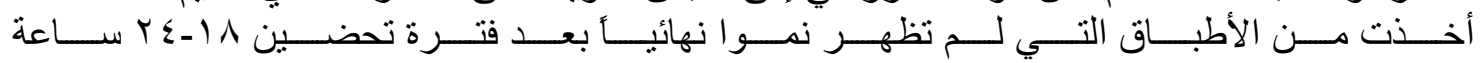
. ( r...0، Iroegbuو Okoil)

\section{النتائج والمناق}

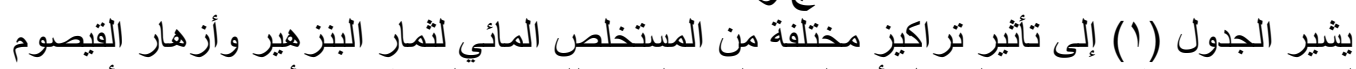

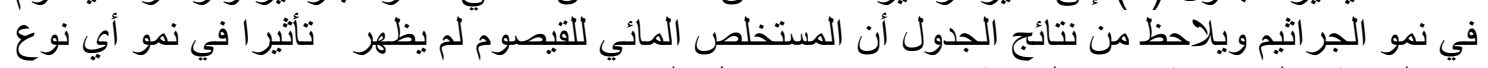

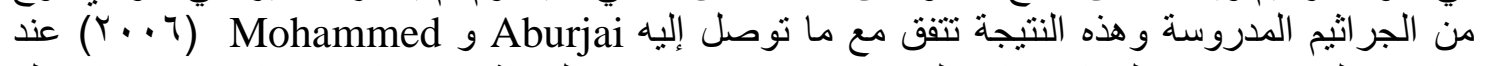

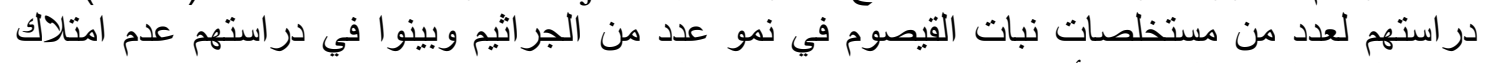

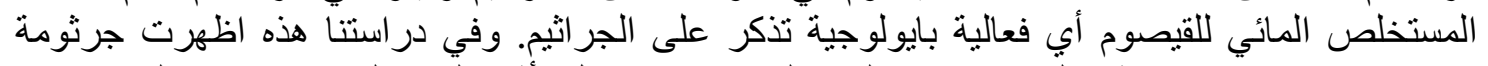
○. Staph. aureus

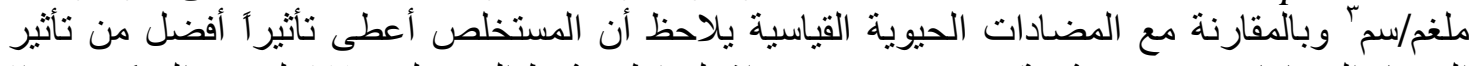

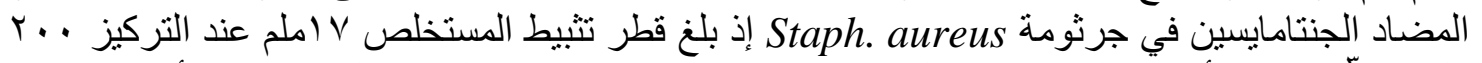

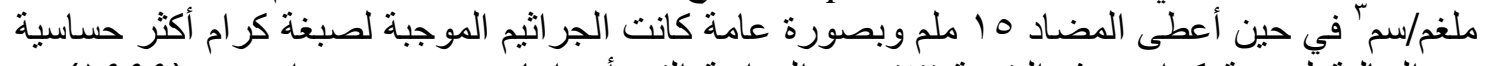

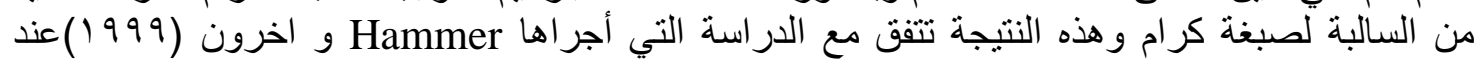

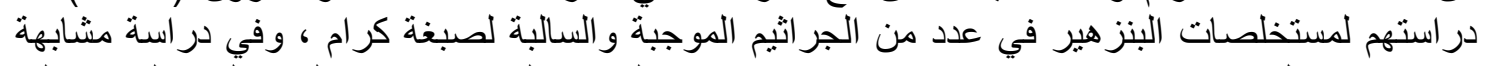

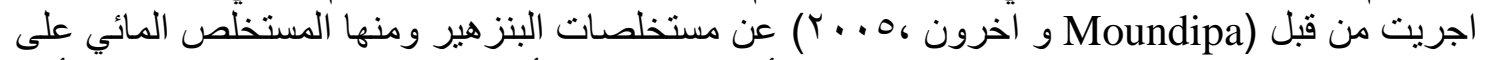

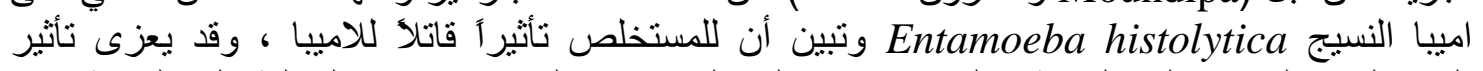

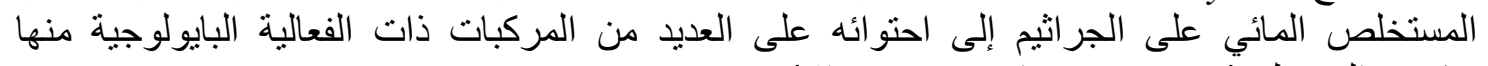

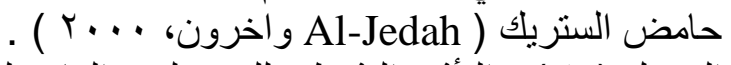
الجدول ( 1 ) : التأثير التثبيطي للمستخلص المائي لنبات القيصوم و البنز هير بتر اكيز مختلفة على الجر اثيم

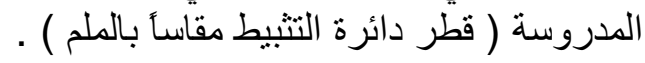




\begin{tabular}{|c|c|c|c|c|c|c|c|c|}
\hline \multicolumn{2}{|c|}{ مقارنه } & \multicolumn{5}{|c|}{ تركيز المستخلص ملغم/سم" } & \multirow{2}{*}{ المعاملة } & \multirow{2}{*}{ العزلات الجرثومية } \\
\hline $\mathrm{TE}$ & $\mathrm{CN}$ & $1 Y .0$ & ro & 0. & $1 \ldots$ & Y.. & & \\
\hline \multirow{2}{*}{ rq } & \multirow{2}{*}{10} & - & - & IT & $1 \varepsilon$ & IV & Cit & \multirow{2}{*}{ Staph. Aureus } \\
\hline & & - & - & - & - & - & Ach & \\
\hline \multirow{2}{*}{$r \varepsilon$} & \multirow{2}{*}{17} & - & - & 11 & $1 \varepsilon$ & 17 & Cit & \multirow{2}{*}{ B.subtilis } \\
\hline & & - & - & - & - & - & Ach & \\
\hline \multirow{2}{*}{ r. } & \multirow{2}{*}{11} & - & - & - & Ir & $1 \varepsilon$ & Cit & \multirow{2}{*}{ Ps.aeruginosa } \\
\hline & & - & - & - & - & - & Ach & \\
\hline \multirow{2}{*}{$r \leq$} & \multirow{2}{*}{19} & - & - & - & 11 & $1 \varepsilon$ & Cit & \multirow{2}{*}{ Pr.vulgris } \\
\hline & & - & - & - & - & - & Ach & \\
\hline \multirow{2}{*}{ rᄉ } & \multirow{2}{*}{ IV } & - & - & - & 11 & $1 \pi$ & Cit & \multirow{2}{*}{ K.pnumonia } \\
\hline & & - & - & - & - & - & Ach & \\
\hline \multirow{2}{*}{ rV } & \multirow{2}{*}{ r) } & - & - & - & Ir & 10 & Cit & \multirow{2}{*}{ E.coli } \\
\hline & & - & - & - & - & - & Ach & \\
\hline
\end{tabular}

- قطر القرص 7 ملم ، ( - ) تثير إلى عدم وجود تأثثر ، Cit البنز هير ، Ach القيصوم . . Tetracycline ( TE ) $30 \mu \mathrm{g} / \mathrm{disk}$, Gentamicin ( CN ) $10 \mu \mathrm{g} / \mathrm{disk}$ -

يبين الجدول (Y) تأثير نر اكيز مختلفة من المستخلص الايثانولي لنبات القيصوم و البنز هير ويلاحظ

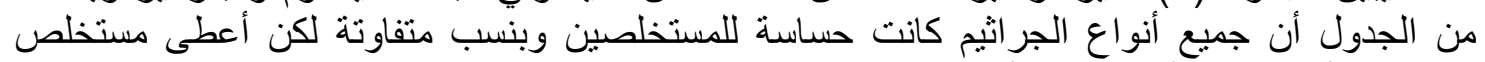

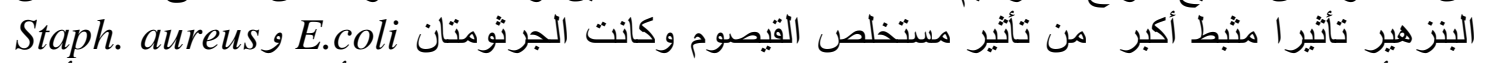

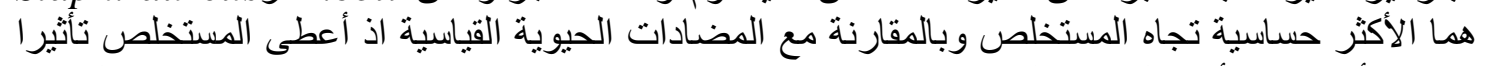

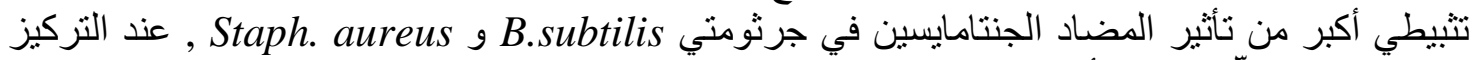

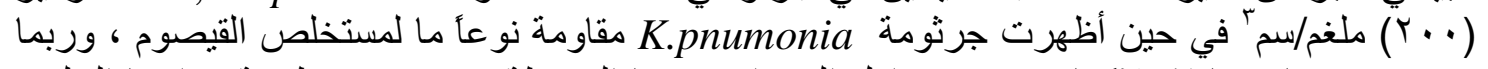

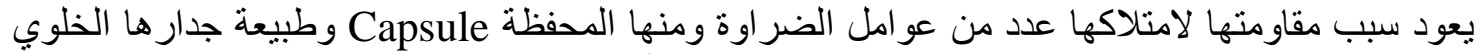

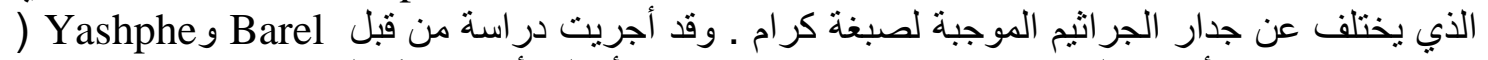

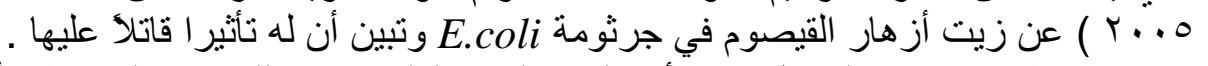

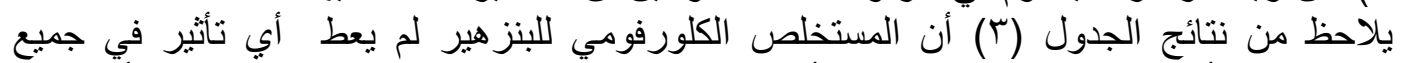

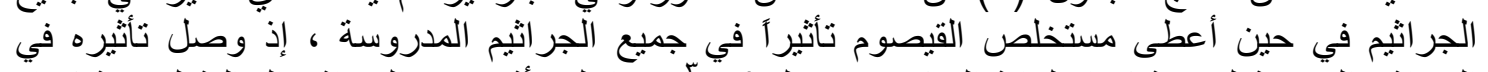

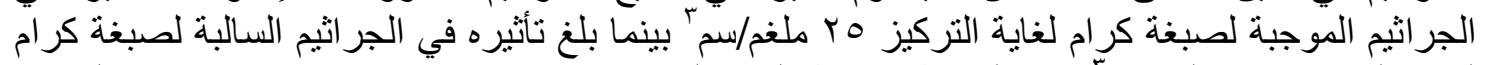

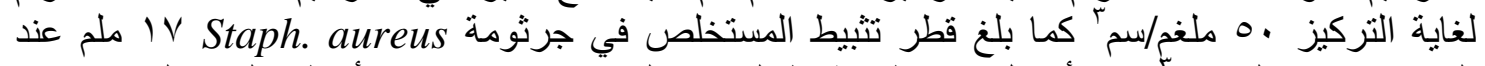

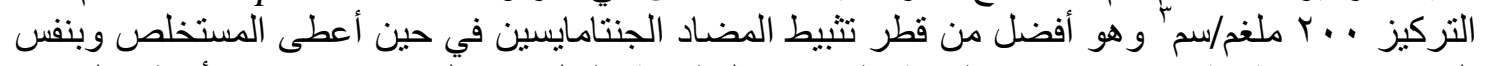

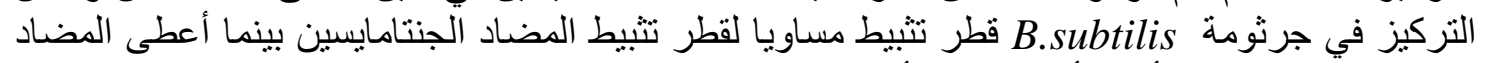

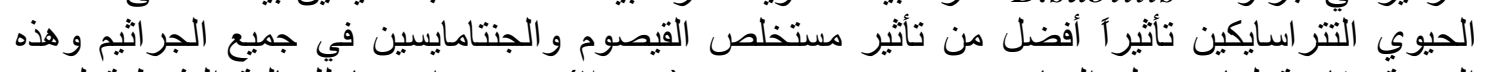

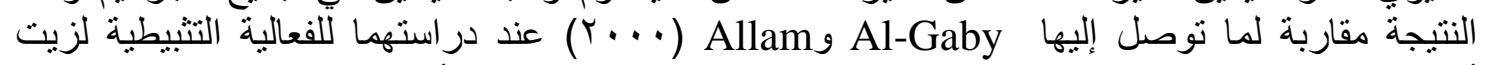

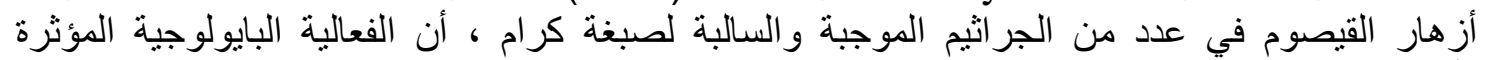

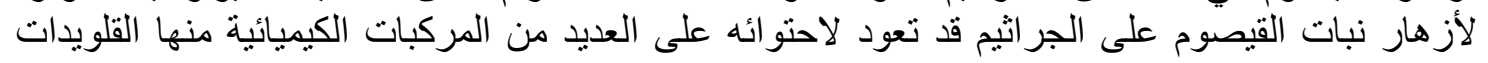
و الفلافوميدات التويدات 
الجدول ( r ) ) : التأثير التنبيطي للمستخلص الايثانولي لنبات القيصوم و البنز هير بتراكيز مختلفة على بلى

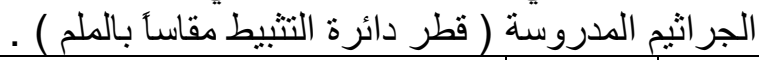

\begin{tabular}{|c|c|c|c|c|c|c|c|c|}
\hline \multicolumn{2}{|c|}{ مقارنة } & \multicolumn{5}{|c|}{ تركيز المستخلص ملغم/سمج } & \multirow{2}{*}{ المعاملة } & \multirow{2}{*}{ العزلات الجرثومية } \\
\hline $\mathrm{TE}$ & $\mathrm{CN}$ & $1 Y .0$ & ro & 0. & $1 \ldots$ & $r \ldots$ & & \\
\hline \multirow{2}{*}{ r } & \multirow{2}{*}{10} & - & IT & $1 \varepsilon$ & 17 & 11 & Cit & \multirow{2}{*}{ Staph. Aureus } \\
\hline & & - & - & IT & $1 \varepsilon$ & IV & Ach & \\
\hline \multirow{2}{*}{$r \leq$} & \multirow{2}{*}{17} & 11 & 14 & 10 & 17 & 11 & Cit & \multirow{2}{*}{ B.subtilis } \\
\hline & & - & 1. & 14 & 10 & 11 & Ach & \\
\hline \multirow{2}{*}{ r. } & \multirow{2}{*}{11} & - & - & IT & $1 \varepsilon$ & IV & Cit & \multirow{2}{*}{ Ps.aeruginosa } \\
\hline & & - & - & 1. & Ir & 17 & Ach & \\
\hline \multirow{2}{*}{$r \leqslant$} & \multirow{2}{*}{19} & - & IY & $1 \varepsilon$ & 17 & 11 & Cit & \multirow{2}{*}{ Pr.vulgris } \\
\hline & & - & - & 1. & Ir & $1 \varepsilon$ & Ach & \\
\hline \multirow{2}{*}{ rᄉ } & \multirow{2}{*}{ iv } & - & - & IT & $1 \varepsilon$ & 17 & Cit & \multirow{2}{*}{ K.pnumonia } \\
\hline & & - & - & - & Ir & 10 & Ach & \\
\hline \multirow{2}{*}{ rV } & \multirow{2}{*}{ r) } & - & IY & $1 \varepsilon$ & 17 & 19 & Cit & \multirow{2}{*}{ E.coli } \\
\hline & & - & - & IT & $1 \varepsilon$ & 17 & Ach & \\
\hline
\end{tabular}

- قطر القرص7 ملم ، ( - ) تشير إلى عدم وجود تأثير ، Cit البنز هير Ach، (

. Tetracycline ( TE ) $30 \mu \mathrm{g} / \mathrm{disk}$, Gentamicin ( CN ) $10 \mu \mathrm{g} / \mathrm{disk}$ -

الجدول (ب ) : التأثير التثبيطي للمستخلص الكلوروفورمي لنبات القيصوم والبنز هير بتر اكيز مختلفة على التى

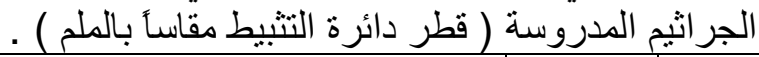

\begin{tabular}{|c|c|c|c|c|c|c|c|c|}
\hline \multicolumn{2}{|c|}{ مقارنه } & \multicolumn{5}{|c|}{ تركيز المستخلص ملغم/سم } & \multirow{2}{*}{ المعاملة } & \multirow{2}{*}{ العز لات الجرثومية } \\
\hline TE & $\mathrm{CN}$ & $1 Y .0$ & ro & 0. & $1 \cdots$ & r.. & & \\
\hline \multirow{2}{*}{ Y } & \multirow{2}{*}{10} & - & - & - & - & - & Cit & \multirow{2}{*}{ Staph. Aureus } \\
\hline & & - & IT & $1 \leqslant$ & 10 & IV & Ach & \\
\hline \multirow{2}{*}{$r \varepsilon$} & \multirow{2}{*}{17} & - & - & - & - & - & Cit & \multirow{2}{*}{ B.subtilis } \\
\hline & & - & 1. & 11 & $1 \varepsilon$ & 17 & Ach & \\
\hline \multirow{2}{*}{ r. } & \multirow{2}{*}{11} & - & - & - & - & - & Cit & \multirow{2}{*}{ Ps.aeruginosa } \\
\hline & & - & - & Ir & $1 \varepsilon$ & IV & Ach & \\
\hline \multirow{2}{*}{$r \varepsilon$} & \multirow{2}{*}{19} & - & - & - & - & - & Cit & \multirow{2}{*}{ Pr.vulgris } \\
\hline & & - & - & IT & $1 \pi$ & 10 & Ach & \\
\hline \multirow{2}{*}{ rA } & \multirow{2}{*}{ iv } & - & - & - & - & - & Cit & \multirow{2}{*}{ K.pnumonia } \\
\hline & & - & - & IY & $1 \pi$ & 17 & Ach & \\
\hline \multirow{2}{*}{$r v$} & \multirow{2}{*}{ r) } & - & - & - & - & - & Cit & \multirow{2}{*}{ E.coli } \\
\hline & & - & - & Ir & $1 \varepsilon$ & IV & Ach & \\
\hline
\end{tabular}

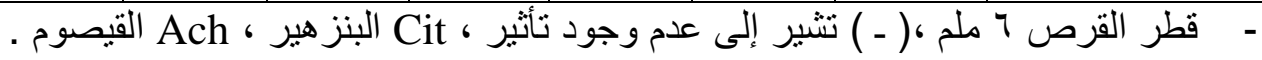

. Tetracycline ( TE ) $30 \mu \mathrm{g} / \mathrm{disk}$, Gentamicin ( CN ) $10 \mu \mathrm{g} / \mathrm{disk}$ -

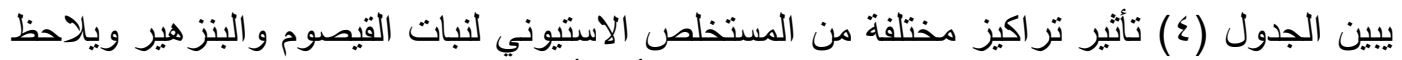

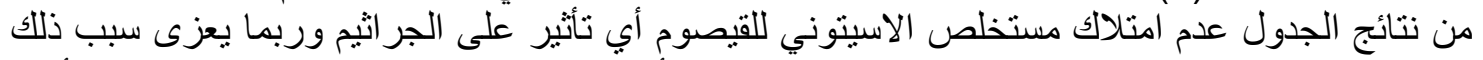

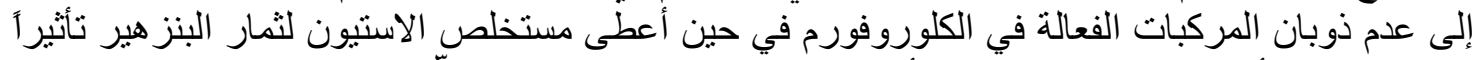

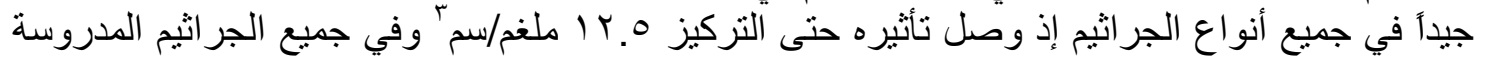




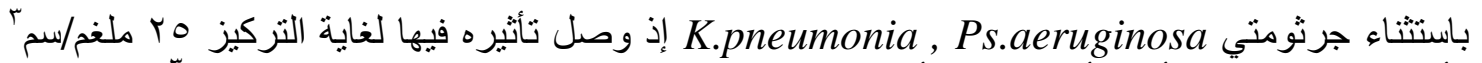

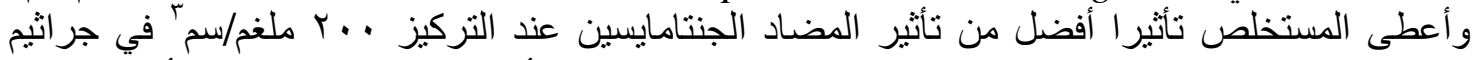

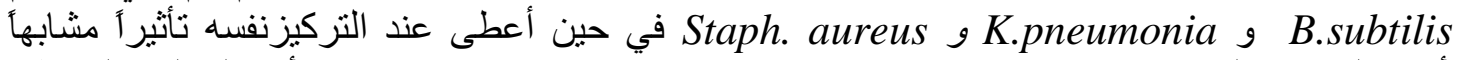

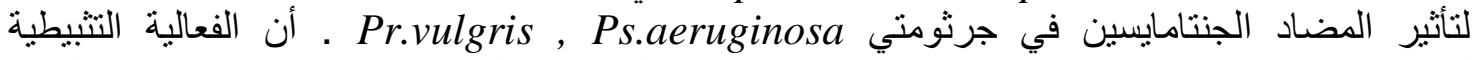

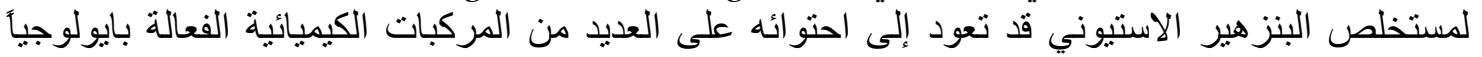

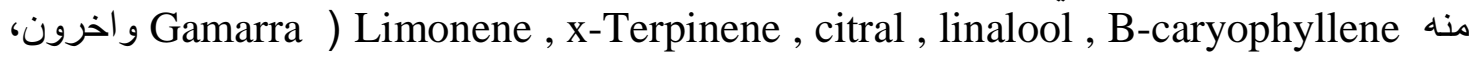
. ( r.. T

الجدول ( ع ) : التأثير التثبطي للمستخلص الاستيوني لنبات القيصوم والبنز هير بتر اكيز مختلفة على

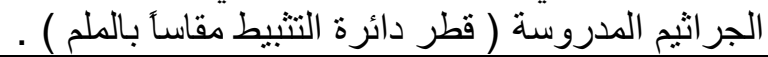

\begin{tabular}{|c|c|c|c|c|c|c|c|c|}
\hline \multicolumn{2}{|c|}{ مقارنه } & \multicolumn{5}{|c|}{ تركيز المستخلص ملغم/سم } & \multirow{2}{*}{ المعاملة } & \multirow{2}{*}{ العزلات الجرثومية } \\
\hline TE & $\mathrm{CN}$ & $1 T_{0}$ & ro & 0. & $1 \ldots$ & $r \ldots$ & & \\
\hline \multirow{2}{*}{ Y } & \multirow{2}{*}{10} & IT & $1 \varepsilon$ & 17 & 11 & $r \cdot$ & Cit & \multirow{2}{*}{ Staph. aureus } \\
\hline & & - & - & - & - & - & Ach & \\
\hline \multirow{2}{*}{$r \leq$} & \multirow{2}{*}{17} & Ir & $1 \leqslant$ & IV & 19 & YI & Cit & \multirow{2}{*}{ B.subtilis } \\
\hline & & - & - & - & - & - & Ach & \\
\hline \multirow{2}{*}{ r. } & \multirow{2}{*}{11} & - & 11 & $1 \varepsilon$ & 17 & 11 & Cit & \multirow{2}{*}{ Ps.aeruginosa } \\
\hline & & - & - & - & - & - & Ach & \\
\hline \multirow{2}{*}{$r \leq$} & \multirow{2}{*}{19} & 11 & 14 & 10 & IV & 19 & Cit & \multirow{2}{*}{ Pr.vulgris } \\
\hline & & - & - & - & - & - & Ach & \\
\hline \multirow{2}{*}{ rA } & \multirow{2}{*}{ iv } & - & 11 & $1 \varepsilon$ & 17 & 11 & Cit & \multirow{2}{*}{ K.pnumonia } \\
\hline & & - & - & - & - & - & Ach & \\
\hline \multirow{2}{*}{ TV } & \multirow{2}{*}{ r) } & IT & $1 \varepsilon$ & 17 & 11 & $r$. & Cit & \multirow{2}{*}{ E.coli } \\
\hline & & - & - & - & - & - & Ach & \\
\hline
\end{tabular}

- قطر القرص 7 ملم ، ( - ) تشير إلى عدم وجود تأثير ، Cit البنز هير ، Ach القيصوم . . Tetracycline ( TE ) $30 \mu \mathrm{g} /$ disk, Gentamicin ( CN ) $10 \mu \mathrm{g} / \mathrm{disk}$ -

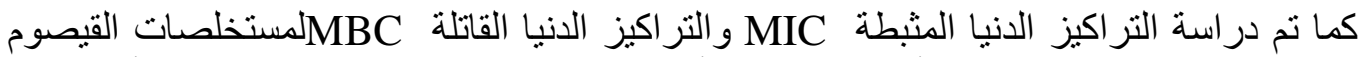

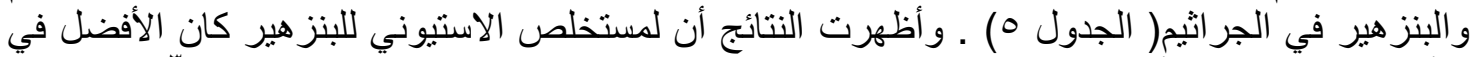

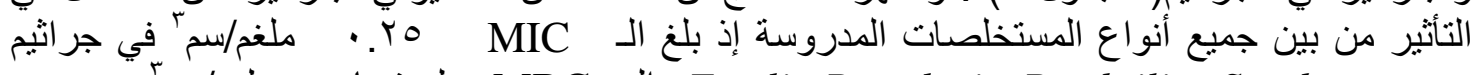
MBC و الـ M.coli, Pr.vulgris, B.subtilis, Staph. aureus

الجدول ( 0 ) : تأثثر التر اكيز الدنيا المثبطة ( MIC ) و التراكيز الدنيا القاتلة ( MBC ) لمستخلصات

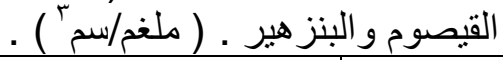

\begin{tabular}{|c|c|c|c|c|c|c|c|c|c|c|c|c|}
\hline \multicolumn{12}{|c|}{ العز لات الجرثومبة } & \multirow{3}{*}{ نوع المعاملة } \\
\hline \multicolumn{2}{|c|}{ E.Coli } & \multicolumn{2}{|c|}{ K.pnuтопia } & \multicolumn{2}{|c|}{ Pr.vulgris } & \multicolumn{2}{|c|}{ Ps.aeruginosa } & \multicolumn{2}{|c|}{ B.subtilis } & \multicolumn{2}{|c|}{ Staph. aureus } & \\
\hline MBC & MIC & MBC & MIC & MBC & MIC & MBC & MIC & MBC & MIC & MBC & MIC & \\
\hline - & 1. & - & 1. & - & $1 . \cdot$ & - & 1. & r.. & $\because 0$ & r.. & $\because 0$ & مستخلص للبنز هير المائي \\
\hline r. & r. ro & r. & .0 & 1. & r ro & r.. & $\therefore 0$ & $1 . \cdot$ & $\therefore$ iro & 1. & $\therefore$ ro & مستخلص للبنز هير الاثينولي \\
\hline r. & .0 & - & $1 . \cdot$ & r.. & $\because 0$ & r.. & $\because 0$ & $1 . \cdot$ & r ro & r. & $\because 0$ & مستخلص للقيصوم الاثينولي \\
\hline r.. & $\because 0$ & r.. & $\because 0$ & r.. & $\because 0$ & r.. & $\because 0$ & r.. & r. ro & r. & r. ro & مستخلص للقيصوم الكلوروفورمي \\
\hline$\because 0$ & $\therefore$ r. & r. & . ro & $\because 0$ & $\cdot$. Iro & $1 . \cdot$ & r. ro & $\because 0$ & . iro & $\because 0$ & $.1 \% 0$ & مستخلص للبنز هير الاستيوني \\
\hline
\end{tabular}




\title{
ANTIBACTERIAL ACTIVITY OF Achillea fragrantissma L. AND FRUITS OF Citrus aurantifolia L. EXTRACTS AGAINST SEVERAL GRAM POSITIVE AND NEGATIVE BACTERIA
}

\author{
Hanan Sami Nori
}

Basic Sci.Dept. , College of Nursing, Mosul Univ. , Iraq.

\begin{abstract}
Biological activities of Citrus aurantifolial and Achillea Fragrantissmal extracts were tested against two species of Gram positive bacteria, Staph. lococcus aureus , Bacillus subtilis and four species of Gram negative bacteria , Pseudomonas aeruginosa, Proteus vulgaris , Kiebsilla pneumonia , Escherichia coli . Fruit of Citrus aurantifoliaL. and flowers of Achillea FragrantissmaL. were extracted using Dist water and Soxhlet apparatus in presence of Ethanol, Chloroform, and Aceton . Water extract of Achillea FragrantissmaL. showed no activity against all bacterial species tested, but the water extract of Citrus aurantifoliaL. showed a good antibacterial activities using disc diffusion method and comparing with the standard antibiotic (Gentamicin and Tetracycline ) . Result also indicated that ethanol extract showed high antibacterial activities in all types species tested . Chloroform extract of Citrus aurantifoliaL. and Acetone extract of Achillea FragrantissmaL.on the other hand, showed no activity on all species of bacteria tested, The study also detected the Minimum Inhibitory Concentration (MIC) and the Minimum Bactericidal Concentration (MBC) of the two plant extracts, tested bacterial showed high sensitivity against acetone extract of Citrus aurantifoliaL. , Staph. aureus, B.subtilis, Pr.vulgris and E.coli was more sensitive than other tested bacteria , the calculated (MIC) reached $0.125 \mathrm{mg} / \mathrm{ml}$ while the ( $\mathrm{MBC}$ ) reached $0.5 \mathrm{mg} / \mathrm{ml}$.
\end{abstract}

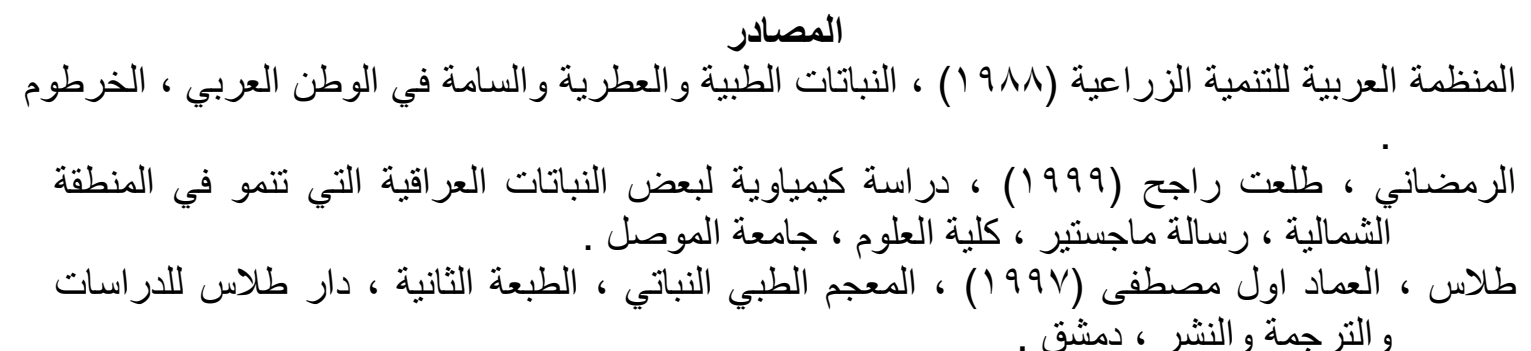

Akinpelu , D.A. and T.M. onakoya ( 2006 ) . Antibacterial activities of medicinal plants used in Folklove-remedies in south western . African.J.Biotech.5(11) : 1076-1081 .

Aburjai , T. and H.Mohammed ( 2006 ) . Antiplatlet, Antibacterial and antifungal activities of Achillea Fakata extracts and evaluation of volatile oil compositionPharma. Mag. 2(7) : 191-198 .

Al-Gaby , A. and R.M. Allam ( 2000 ) . Chemical Analysis , antimicrobial activity and essential oils from some wild herbs in Egypt . H. Herbs. Medplants . $7(1): 15-29$. 
Al-Jedah , J.H. , M.Z. Ali and R.K. Robinson ( 2000 ). The inhibitory action of spices against pathogens that might be capable of growth in fish sause ( mehiawah ) from the middle east . Inter.J.Food micro. $57: 129-133$.

Al-Nowaihi , A.M. , A.M.A. Osman , A. Raziek and T.MEL-Lamey ( 2005 ) . Effect of some ecological factors on the chemical compounds in some xerophytes for enchancing their use . J. Environ. Sci. 10(2) : 373 .

Barel , S. and J.Yashphe ( 2005 ) . Effect of essential oil from Achillea Fragrantissima on Escherichia coli-current . Micro. 19(6) : 337-341 .

Bauer , A. , W.A.M. Kirbay , J.S. Sherris and M. Turk ( 1966 ) . Antibiotic susceptibility testing by astandardized single disk method. Am.J.Clin.Pathol. 45 : 493-496.

Ellof , J.N. , J.O. Famakin and D.R.P. Katerer ( 2005 ). Isolation of an antibacterial stilbene from combretum woodii ( Combretaceae ) Leaves . African.J.Biotech. 4(10): 1167-1171 .

Farombi , E.O ( 2003 ) . African indigenous plants with chemotherapeutic potentials and biotechnological approach to the production of bioactive prophylactic agents . A.J.B. 2(12) : 662-671 .

Gamarra , F.M. , L.S. Saknaka , E.B. Tambourgi and F.A. Eabral ( 2006 ) . Influnce on the quality of essential lemon ( Citrus aurantifolia) oil by distillation process . Braz . J.Chem.Eng.23(1) : 147-151 .

Hammer , K.A. , C.F. Carson and T.V. Riley ( 1999 ). Antimicrobial activity of essential oils and other plant extracts . J.Applied Micro. 86: 985-990 .

Koneman, E.W ; S.D. Allen ; W.M. Janda ; P.C. Schreckenbergev and W.C.Win (1997).Color Atlas and text book of diagnostic microbiology. $5^{\text {th }}$ ed, J.B. Lippincott Raven Publishers,Philadelphia

Moundipa , P.F , G.M. Kamini , and C.B. Iris ( 2005 ) . Invetro Amoebicidal activity of some medicinal plants of the Bamun Region ( Cameroon ). A.J.Trad.Cam. 2(2) : 113-121 .

Okoil , S. and C.Iroegbu ( 2005 ). Invitro antibacterial activity of Synclisa scabrida whole root extracts . African.J.Biotech. 4(9) : 946-952 .

Riose , J.L, M.C. Recio and A. Villar ( 1987 ) . Antimicrobial activity of selected plants employed in the Spanish Mediterranean area . Ethnopharmacol . 21 : 139-152.

Saliva , O. , A. Duarte , S. Viegas , H. Barroso , J. Mchado , I. Pires , J. Cabrita and E. Gomes ( 1997 ) . Antimicrobial activity of Terminalia Macroptera root . J. Ethnopharmacol . $57:$ 203-207 .

Waage , S.K. and P.A. Hedin ( 1985 ) . Quercetin 3-O-Galactosyl ( 1-6 ) Glucoside acompound from narrow leafleted with antimicrobial activity Phytochemistry . 24: 243-245 . 